\title{
In vivo non-viral gene delivery of human vascular endothelial growth factor improves revascularisation and restoration of euglycaemia after human islet transplantation into mouse liver
}

\author{
M. Shimoda • S. Chen • H. Noguchi • S. Matsumoto • \\ P. A. Grayburn
}

Received: 6 January 2010/Accepted: 3 March 2010 /Published online: 20 April 2010

(C) Springer-Verlag 2010

\begin{abstract}
Aims/hypothesis Delivery of the gene for human vascular endothelial growth factor ( $V E G F$, also known as VEGFA) to both the transplanted islets and the surrounding tissue may promote islet revascularisation and survival. We previously showed the effective delivery of $V E G F$ gene to rat myocardium by an ultrasound-mediated gene-transfer method named ultrasound-targeted microbubble destruction (UTMD). Here we examined the effect of non-viral $V E G F$ delivery using UTMD on transplanted islets in vivo.

Methods A marginal number of human islets were transplanted into livers of mice which were a model for diabetes. Then, non-viral plasmid vectors encoding VEGF (VEGF group, $n=11)$ or the gene for green fluorescent protein $(G F P)$ (GFP group, $n=7$ ) were introduced into the host liver by UTMD. Transplantation without gene delivery was performed as a control (no-UTMD group, $n=8$ ). Blood glucose, serum human insulin, C-peptide levels and the revascularisation in graft islets were evaluated.

Results Restoration of euglycaemia occurred in $13 \%$ in the no-UTMD group and $14 \%$ in the GFP group, whereas $73 \%$ mice in the VEGF group became euglycaemic at day 30 $(p<0.05$ in no-UTMD vs VEGF). Serum human insulin and C-peptide were significantly higher in the VEGF group at
\end{abstract}

day 32 (insulin: no-UTMD, 17 \pm 8 ; GFP, 37 \pm 17 ; VEGF, $109 \pm 26 \mathrm{pmol} / \mathrm{l}$, respectively, $p<0.05$; C-peptide: no-UTMD, $68 \pm 38$; GFP, $115 \pm 58$; VEGF, $791 \pm 230 \mathrm{pmol} / \mathrm{l}$, respectively, $p<0.05$ ). Vessel density in graft islets was significantly higher in the VEGF group (no-UTMD, 169 \pm 36 ; GFP, $227 \pm$ 39; VEGF, $649 \pm 51$ counts $/ \mathrm{mm}^{2}$, respectively, $p<0.05$ ).

Conclusions/interpretation Delivery of VEGF gene to host liver using UTMD promoted islet revascularisation after islet transplantation and improved the restoration of euglycaemia.

Keywords Gene therapy · Human vascular endothelial growth factor Islet transplantation .

Non-viral gene delivery $\cdot$ Revascularisation
Abbreviations
ALT Alanine aminotransferase
AST Aspartate aminotransferase
GFP Green fluorescent protein
VEGF Human vascular endothelial growth factor
IPGTT Intraperitoneal glucose tolerance test
pDNA Plasmid DNA
STZ Streptozotocin
UTMD Ultrasound-targeted microbubble destruction

M. Shimoda $\cdot$ S. Chen $\cdot$ P. A. Grayburn $(\bowtie)$

Division of Cardiology, Department of Internal Medicine, Baylor University Medical Center, Baylor Heart and Vascular Institute, 621 North Hall St, Suite H030,

Dallas, TX 75226, USA

e-mail: paulgr@baylorhealth.edu

M. Shimoda $\cdot$ H. Noguchi $\cdot$ S. Matsumoto Baylor Research Institute Fort Worth Campus, Fort Worth, TX, USA

\section{Introduction}

Islet transplantation is a promising treatment for type 1 diabetes; however, efficacy of transplantation needs to be improved, because currently multiple transplantation is required to achieve insulin-free status [1,2]. It was shown that gene delivery to islets can improve the function and 
survival of islets [3-6], but all previous studies used viral vectors. A viral vector has high efficacy for delivering genes, but adverse events have been related to enhancermediated mutagenesis of genomic DNA [7] or immunological responses to viral proteins [8].

In contrast, delivery of naked plasmid DNA (pDNA) does not transport toxic or immunogenic viral protein or polymer particles in vivo. However, plasmid vectors have been limited by low transfection efficiency. To obtain high gene expression with pDNAs, we have established a novel ultrasound-mediated gene-transfer method known as ultrasound-targeted microbubble destruction (UTMD). Microbubbles, which consist of a lipid shell encapsulating perfluorocarbon gas, are injected into the circulation. The pDNAs are incorporated into the lipid shell. Under ultrasound exposure, the microbubbles burst and create transient pores in the membranes of surrounding cells, and pDNAs are inserted into the cells. UTMD has many desired characteristics for gene therapy including low toxicity, low immunogenicity, potential for repeated application, organ specificity and broad applicability to acoustically accessible organs. We previously demonstrated that delivery of the gene $(V E G F)$ for human vascular endothelial growth factor (hVEGF) to rat myocardium by UTMD resulted in significant increases in myocardial capillary and arteriolar density [9]. Moreover, we reported that our UTMD technique enabled us to effectively deliver pDNAs to rat pancreatic beta cells in vivo $[10,11]$. Lack of intra-islet microvasculature is one of the most important factors in loss of graft islets. Therefore, we hypothesise that early loss of islet grafts could be attenuated by delivery of the VEGF gene to promote revascularisation.

In this study, we used a transplantation model in which human islets were transplanted into mouse liver via the portal vein. This model is similar to the clinical setting. $V E G F$ gene was delivered to the host liver by UTMD to examine whether it could facilitate revascularisation and improve the survival and function of the transplanted islets.

\section{Methods}

Human islets Seven donor pancreases were procured from deceased multiorgan donors after obtaining consent for research through the local Organ Procurement Organizations (Southwest Transplant Alliance, Dallas, TX, USA; LifeGift, Fort Worth, TX, USA). Baylor Regional Transplant Institute Surgeons using the standardised surgical technique performed pancreas procurement [12]. Pancreases were preserved using the ductal injection method with ET-KYOTO solution (Otsuka Pharmaceutical Factory, Naruto, Japan) followed by a two-layer method until the islet isolation procedure
[12]. Islet isolation was performed using the modified Ricordi method [13]. The donor and isolation variables were as follows: donor age $35.1 \pm 5.6$ years; two men and five women; BMI $28.0 \pm 1.5 \mathrm{~kg} / \mathrm{m}^{2}$; cold ischaemic time $171 \pm$ $21 \mathrm{~min}$; purity $77 \pm 5 \%$; viability $97 \pm 1 \%$; stimulation index $10.4 \pm 3.4$. The isolated islets were preserved in CMRLsupplemented culture medium (Cellgro, Manassas, VA, USA) containing 10\% (vol./vol.) FBS (Atlanta Biologicals, Lawrenceville, GA, USA) at $4^{\circ} \mathrm{C}$ overnight. A total of 500 islets of similar size (approximately $200 \mu \mathrm{m}$ ) were handpicked for transplantation. Our preliminary experiment showed $10-20 \%$ of mice became euglycaemic after transplantation of 500 islets. Therefore, we determined the number as a marginal.

Plasmid constructs Plasmids expressing the $V E G F_{165}$ (also known as NRP2) gene under an enhanced CMV promoter (pCI-hVEGF) were made as follows: cDNA of $V E G F_{165}$ was obtained as previously described [9]. Briefly, a fulllength cDNA of $V E G F_{165}$ obtained from a healthy volunteer's blood was PCR amplified by using the following PCR primers that contain a restriction site (the restriction sites are underlined): primer 1 (Xhol) 5'-TTCCTCGAGAATGA ACTTTCTGCTGCTGTCTTG-3'; primer 2 (SmaI) 5'AAACCCGGGTCACCGCCTCGGCTTGTCA-3'. The DNA was digested with XhoI and SmaI and then ligated into the corresponding sites of pCI mammalian expression vector (Promega, Madison, WI, USA). The plasmids were sequenced to confirm that no artefactual mutations were present. Plasmids encoding green fluorescent protein gene $(G F P)$ under the enhanced CMV promoter (pCS2-GFP) were used as a control.

Manufacture of plasmid-containing lipid-stabilised microbubbles Lipid-stabilised microbubbles were prepared as previously described in our laboratory [10]. Briefly, a total of $200 \mu \mathrm{l}$ DPPC (1,2-dipalmitoyl-sn-glycero-3phosphatidylcholine; Sigma, St Louis, MO, USA, 2.5 $\mathrm{mg} / \mathrm{ml}$ ) and DPPE (1,2-dipalmitoyl-sn-glycero-3-phosphatidylethanolamine; Sigma, $0.5 \mathrm{mg} / \mathrm{ml}$ ) solution, $5 \mu 110 \%$ (wt/vol.) human albumin and $50 \mu \mathrm{l}$ pure glycerol were added to $1.5 \mathrm{ml}$ vials containing $2 \mathrm{mg}$ plasmids dissolved in $50 \mu 1$ Lipofectamine $^{2000}$ (Invitrogen, Carlsbad, CA, USA) and mixed well and incubated at room temperature for $10 \mathrm{~min}$, the remaining headspace was filled with the perfluoropropane gas (Air Products, Allentown, PA, USA) and then mechanically shaken for $30 \mathrm{~s}$ by a dental amalgamator (Vialmix; Bristol-Myers Squibb Medical Imaging, N. Billerica, MA, USA). The mean diameter and concentration of the microbubbles were measured by a particle counter (Beckman Coulter Multisizer III; Fullerton, CA, USA). Ten microlitres of microbubble solution, which consisted of approximately $50 \mu \mathrm{g}$ of the plasmids, was 
diluted with $0.3 \mathrm{ml}$ PBS just before the injection and injected into each mouse.

Animal protocol and UTMD Animal studies were performed in accordance with National Institutes of Health (NIH) recommendations and the approval of the institutional animal care and use committee. Streptozotocin (STZ, $150 \mathrm{mg} / \mathrm{kg}$ ) was administered to male nude mice (8- to 13-week-old; Harlan, Houston, TX, USA) by i.p. injection. The mice were considered to be diabetic after two consecutive measurements of blood glucose $\geq 19.4 \mathrm{mmol} / \mathrm{l}$. Pre-transplantation diabetic status was not different among three groups (duration of diabetes: $4 \pm 1$ day, pre-transplant blood glucose: $24.6 \pm 0.6 \mathrm{mmol} / \mathrm{l})$. Mice were anaesthetised with i.p. ketamine $(100 \mathrm{mg} / \mathrm{kg})$ and xylazine $(10 \mathrm{mg} / \mathrm{kg})$. The ileo-caecal vein (a branch of the portal vein) was pulled out by small midline section onto the lower abdomen so that the ultrasound probe could be put on the upper abdomen (Fig. 1a). A 27 G wing needle was inserted and fixed with a haemoclip (Fig. 1b). In this study, 26 mice received one of three treatments: (1) human islet transplantation alone without UTMD (no-UTMD group, $n=8$ ); (2) human islet transplantation and UTMD with plasmids encoding GFP gene (GFP group, $n=7$ ); (3) human islet transplantation and UTMD with plasmids encoding $V E G F_{165}$ (VEGF group, $n=11$ ).

Hand-picked human islets $(n=500)$ were transplanted into the mouse liver through the ileo-caecal vein for each mouse. Then each mouse was randomly assigned to one of three groups. Microbubbles with plasmids were infused immediately after islet transplantation through the ileo- caecal vein in the GFP and VEGF groups for 60-90 s via a pump (Genie; Kent Scientific, Torrington, CT, USA).

During the infusion, ultrasound was directed to the liver using a commercially available ultrasound transducer (S3, Sonos 5500; Philips Ultrasound, Bothell, WA, USA). Ultrasound was then applied in ultraharmonic mode (transmit 1.3 MHz/receive 3.6 MHz) at a mechanical index of 1.4. Four bursts of ultrasound were triggered to every fourth end-systole by an electrocardiogram using a delay of $85 \mathrm{~ms}$ after the peak of the $\mathrm{R}$ wave. These settings have been shown to be optimal for plasmid delivery by UTMD using this instrument [9-11]. Bubble destruction was visually apparent in all mice (Fig. 1c, d). After infusion, the animals were allowed to recover. To study the efficacy of UTMD, the technique was performed for normal nude mice as described above without islet transplantation $(n=3)$. They were killed at 1-28 days after treatment for measurement of serum hVEGF levels with an ELISA kit (ALPCO Diagnostics, Salem, NH, USA). At day 3, the liver, pancreas, spleen, kidneys, stomach, lungs and heart were harvested for the assessment of gene expression by RT-PCR.

To evaluate the extent and area of hVEGF production in the transplanted islets, we transplanted islets into normal nude mice via the portal vein and then consecutively performed UTMD with VEGF plasmids. Three days after the treatment, the liver was harvested and examined by immunohistochemistry.

Effect of UTMD on liver To evaluate liver toxicity and unwanted effects of hVEGF such as excessive liver angiogenesis and uncontrolled liver growth, UTMD with
Fig. 1 UTMD to mouse liver via the ileo-caecal vein. $\mathbf{a}, \mathbf{b}$ Setup of UTMD. An ultrasound probe was put on the upper abdomen. Arrow, probe. c, d Images of ultrasound. c Mouse liver without microbubbles. The liver was displayed at low echo level. d Mouse liver after injecting microbubbles. White opacification was detected before microbubble destruction (left), and it disappeared after destruction (right)
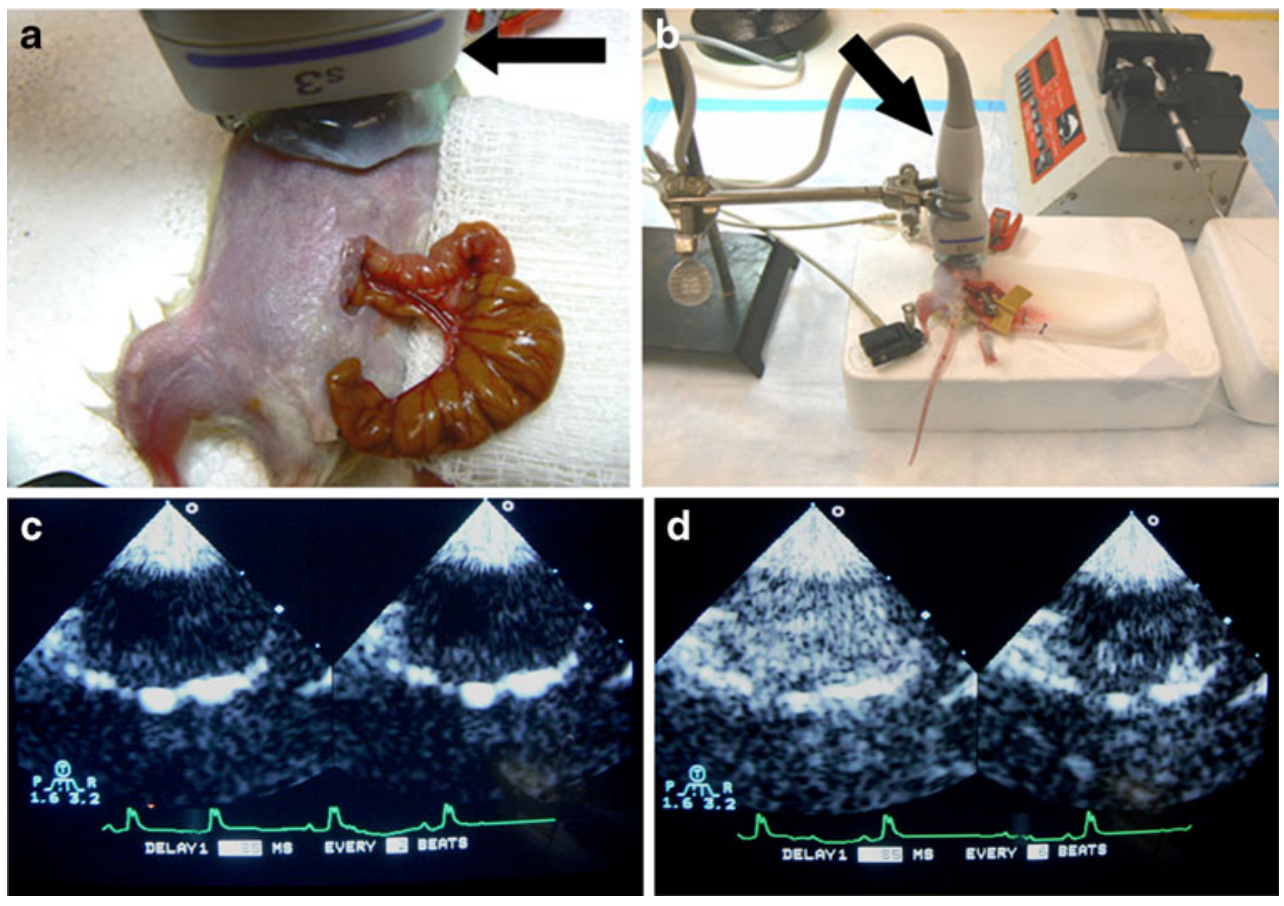
$V E G F$ plasmid via the portal vein was performed as described above. The blood was collected at different time points after UTMD. Samples were analysed for the presence of alanine aminotransferase (ALT) and aspartate aminotransferase (AST) by enzyme assay kits (Thermo Scientific, Waltham, MA, USA). Histological examination was performed after haematoxylin and eosin staining of formalin-fixed mouse liver sections isolated at 2, 7 and 30 days after UTMD.

Assessment of treated mice Non-fasting glucose levels of each mouse were measured with blood glucose test strips (Precision Xtra; Abbott Diabetes Care, Alameda, CA, USA) up to 30 days after transplantation. When two consecutive blood glucose level measurements were $<11.1 \mathrm{mmol} / \mathrm{l}$, restoration of euglycaemia was considered to be present. Recurrence of diabetes was defined as return of the blood glucose to $>11.1 \mathrm{mmol} / \mathrm{l}$. At day 31, i.p. glucose tolerance testing (IPGTT) was performed. After overnight fasting, glucose $(2 \mathrm{~g} / \mathrm{kg})$ was injected i.p. The blood glucose levels were measured for $120 \mathrm{~min}$ after injection. Animals were killed at day 32. The harvested liver was weighed and examined by immunohistochemistry. The blood was collected for measuring human insulin and human C-peptide levels with ELISA kits (ALPCO).

$R T-P C R$ The tissue samples were frozen in liquid nitrogen and stored at $-80^{\circ} \mathrm{C}$. Total RNA was prepared from TRIzol (Invitrogen) according to the manufacturer's instructions and was reverse transcribed using a SuperScript III FirstStrand Synthesis System (Invitrogen). PCR was performed in $20 \mu \mathrm{l}$ volume containing $1 \mu \mathrm{l}$ cDNA, $10 \mu \mathrm{l}$ HotStarTaq Master Mix (Qiagen, Valencia, CA, USA) and 10 pmol of each primer: GAPDH for human and Gapdh for mouse: 5'CCCTTCATTGACCTCAACTACATG-3'(sense); 5'TTCCATTGATGACAAGCTTCCC-3'(antisense); VEGF: 5'-AAGGAGGAGGGCAGAATCAT-3'(sense); 5'-ATCTG CATGGTGATGTTGGA-3'(antisense); mouse Vegf: 5'ACGACAGAAGGAGAGCAGAAGT-3'(sense); 5'-CA TGGTGATGTTGCTCTCTGAC-3'. The PCR conditions included denaturation at $95^{\circ} \mathrm{C}$ for $5 \mathrm{~min}$, followed by $25-35$ cycles of amplification by sequential denaturation at $94^{\circ} \mathrm{C}$ for $30 \mathrm{~s}$ and primer annealing as well as strand extension for $1 \mathrm{~min}$. The RT-PCR products were then analysed on $2 \%$ (wt/vol.) agarose gels.

Immunohistochemistry The collected tissues were fixed in $4 \%$ (wt/vol.) paraformaldehyde at $4{ }^{\circ} \mathrm{C}$ overnight and equilibrated in $30 \%$ (wt/vol.) sucrose at $4{ }^{\circ} \mathrm{C}$ overnight for cryoprotection. The tissues were cryopreserved with Tissue Tek optimal cutting temperature (OCT) compound (Sakura Finetek, Torrance, CA, USA) at $-80^{\circ} \mathrm{C}$. Sections were cut at $10 \mu \mathrm{m}$ increments and placed onto positively charged microscope slides. Sections were permeabilised with $0.1 \%$ (vol./vol.) Triton X-100 for $3 \mathrm{~min}$ and incubated for $30 \mathrm{~min}$ in $20 \%$ (wt/vol.) Aquablock (East Coast Biologics, North Berwick, MA, USA) for blocking. Antibodies to the following antigens were used: guinea pig anti-human insulin (1:200; Abcam, Cambridge, MA, USA), rabbit anti-glucagon (1:20; Millipore, Billerica, MA, USA), goat anti-vimentin (1:10; Sigma-Aldrich, St Louis, MO, USA), mouse anti-human CD31 (1:50; BD Biosciences, San Jose, CA, USA), rat anti-mouse CD31 (1:100; BD Biosciences) and mouse anti-hVEGF (1:100; Millipore). The sections with these first antibodies were incubated at $4^{\circ} \mathrm{C}$ overnight. The antigens were visualised using appropriate secondary antibodies conjugated with FITC, Cy3 (Jackson ImmunoResearch Laboratories, West Grove, PA, USA), Alexa-fluor488 and Alexa-fluor-568 (Invitrogen). Secondary antibodies were used at concentrations recommended by the manufacturer. Then, DAPI was added to the sections for nuclear staining. In this study, the second antibodies against human CD31 and mouse CD31 were labelled with the same fluorochrome. Islets were visualised on a fluorescence microscope and images were analysed using MetaMorph software (Molecular Devices, Sunnyvale, CA, USA).

Insulin-positive beta cell mass in the host liver (left lobe) at day 32 after treatment was measured as previously described [14].

Capillary density measurement Intra-insular CD31-positive vessels visualised by immunohistochemistry were considered as capillaries. The capillaries were counted by the use of fluorescence microscopy at a magnification of $\times 200$. At least two photomicrographs of the insulin-positive graft islets in the host liver were taken for each mouse. The area of each islet was measured with ImageJ software (National Institutes of Health, Bethesda, MA, USA). Pre-isolation human islets were assessed as a control. Capillary density was expressed as the number $/ \mathrm{mm}^{2}$. The investigator reading the capillary density was blinded to treatment group. The vessel density of mouse liver was measured with a similar procedure.

Effect of hVEGF on endogenous pancreas To investigate the effect of circulating hVEGF on endogenous pancreas, UTMD with $V E G F$ plasmid to the liver was performed in STZ-induced diabetic mice. Normal mice and STZ-induced diabetic mice were used as controls. Non-fasting blood glucose levels were measured for 20 days. At day 20 after UTMD, the pancreas in each group was harvested and investigated with immunohistochemistry for insulin (beta cells) and CD31 (capillaries). Insulin-positive beta cell mass in endogenous pancreas was measured as previously described [14]. The vessel density in the islets was measured as described above. 
Statistical analysis Data are expressed as means \pm SEM. Statistical significance of the differences among the three groups was determined by ANOVA followed by Student's $t$ test with Bonferroni correction. Differences of the ratios among the three groups were analysed by Tukey's honestly significant difference test.

\section{Results}

Effect of UTMD on gene delivery to liver via the portal vein UTMD with $\mathrm{pCI}$-hVEGF and $\mathrm{pCS} 2-\mathrm{GFP}$ plasmids was applied to the liver of mice with intraportal injection of the microbubbles. At day 3, hVEGF or GFP production was detected in the liver (Fig. 2). According to immunohistochemical analysis, hVEGF was strongly produced near portal veins, but the production was distributed in a patchy fashion throughout the liver (Fig. 2a-c), whereas nontreated liver did not produce hVEGF (Fig. 2d). GFP production was detected in GFP-induced mouse liver (Fig. 2e). RT-PCR analysis showed that mouse Vegf was expressed in normal mouse liver; however, human $V E G F$ was never expressed. On the other hand, in the $h V E G F$ induced mice, $V E G F$ expression was clearly detected (Fig. 2f).

Serum hVEGF level after UTMD Our preliminary experiments using pCI-hVEGF in vitro proved that hVEGF protein was secreted from the transfected cells into culture media (data not shown). We evaluated hVEGF levels in the blood of the mice treated with UTMD. hVEGF was detected for 14 days after UTMD, although the levels were low (Fig. 2g). This might be because of the effect of dilution and decomposition by blood.

Organ specificity of gene delivery Various organs were harvested from the mice 3 days after UTMD via the portal vein and the $V E G F$ expression in each organ was examined by RT-PCR. VEGF was strongly expressed in the liver, whereas other organs showed no expression (Fig. 2h). The right kidney slightly expressed $V E G F$, probably because it was exposed to ultrasound because of its anatomical proximity to liver.
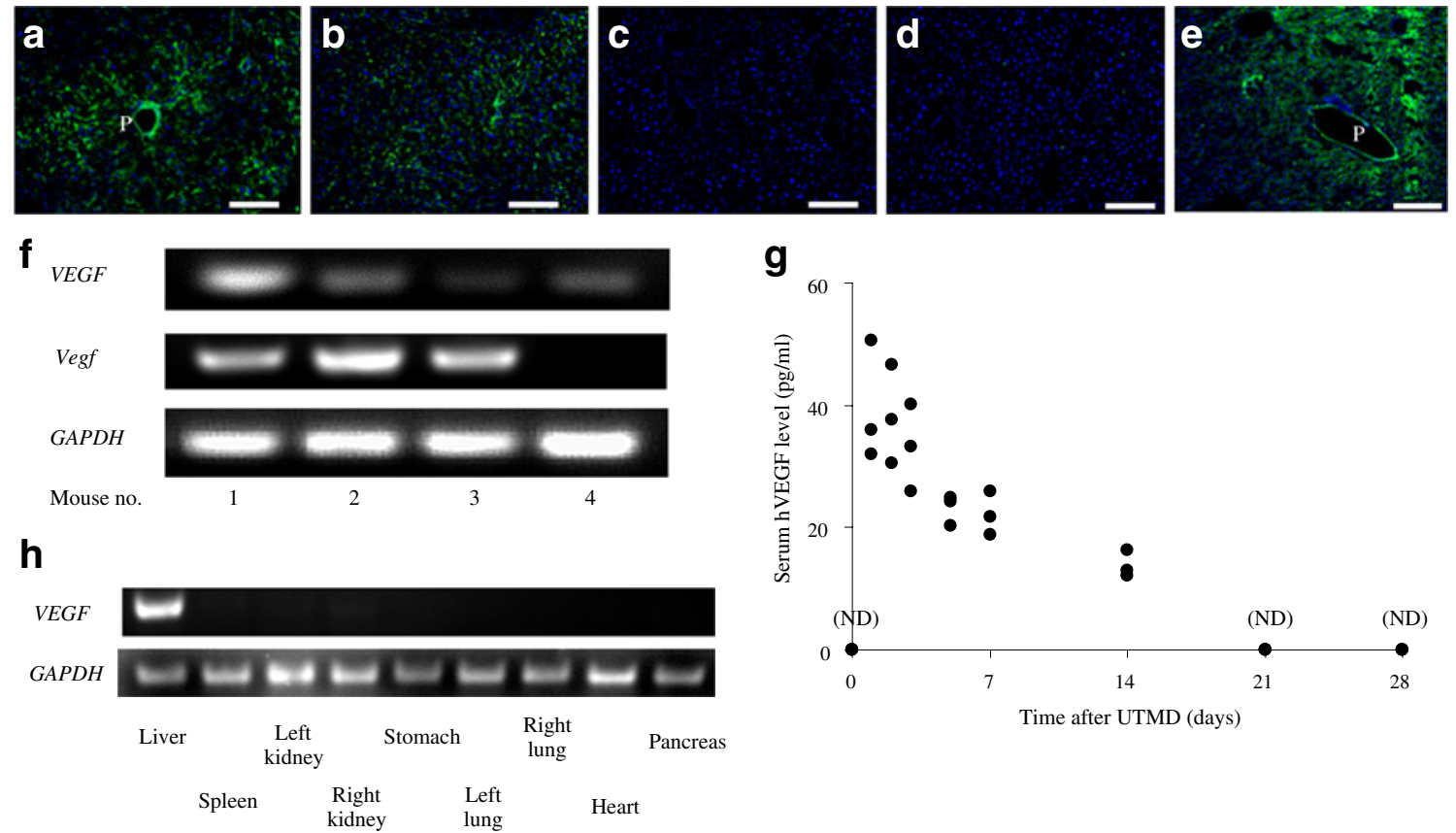

g

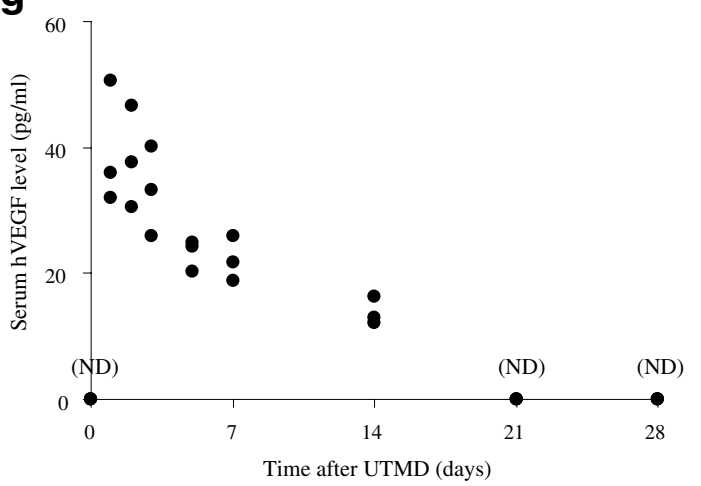

Fig. $2 V E G F$ gene delivery by UTMD. Mouse liver was removed after UTMD with $V E G F$ or $G F P$ plasmid and examined by immunohistochemistry and RT-PCR. a-e Immunohistochemical analysis of mouse liver 3 days (a), 10 days (b) and 14 days (c) after UTMD with $V E G F$. a, b hVEGF production was detected, especially near a portal vein. c hVEGF levels became very low. d Normal mouse liver as a control. hVEGF was not produced. e GFP-induced mouse. GFP production was detected. P, portal vein; green, VEGF (a-d) or GFP (e); blue, DAPI. Magnification $\times 100$. Scale bar, $100 \mu \mathrm{m}$. f RTPCR of VEGF-UTMD mouse liver. $V E G F$ was strongly expressed in

the UTMD-treated group (lanes 1-3), whereas it was not detected in the non-transfected control (lane 4). All mice expressed endogenous mouse Vegf. Gapdh was used as standard. g Serum hVEGF level of UTMD-treated mice $(n=3)$. hVEGF was detected up to 14 days after UTMD. ND, not determined. h Organ specificity of $V E G F$ expression. Various organs were harvested 3 days after UTMD with $V E G F$ plasmid and $V E G F$ expression was examined by RT-PCR. $V E G F$ expression was strongly detected in liver, whereas it was hardly expressed in other organs. Slight expression was seen in right kidney, because this organ was exposed to ultrasound anatomically 
Effect of UTMD and hVEGF on liver The toxicity in the mouse livers caused by UTMD was examined. Serum ALT and AST levels were measured after UTMD. Both ALT and AST levels were slightly elevated at the first day after UTMD and then rapidly declined to normal levels (Fig. 3a). These data indicated that toxicity was trifling and transient. To further determine the extent of hepatic injury following UTMD, the livers were histologically examined at 2, 7 and 30 days after UTMD. Although very slight disruption of hepatocyte architecture was observed at day 2 , the majority of the hepatic parenchyma did not show significant histological abnormality (Fig. 3b).

To examine whether local expression of $V E G F$ could increase the vessels in liver or cause uncontrollable angiogenesis and liver growth, the vessel density in liver and the weight of liver (left lobe) were measured. There were no remarkable differences among the three groups (no-UTMD, GFP and VEGF, Fig. 3c, d).

Effect of circulating hVEGF on endogenous pancreas As shown in Fig. 2, there was no hVEGF production in the pancreas after UTMD, but hVEGF was detected in serum. We investigated whether this low level of hVEGF could affect endogenous pancreatic beta cells and islet vasculature. VEGF gene delivery to liver did not influence blood glucose levels (Fig. 3e), endogenous beta cell mass and vessel density in islets (Fig. 3f-h) for STZ-induced diabetic mice. a

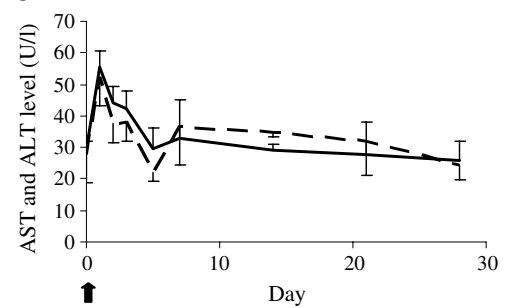

C

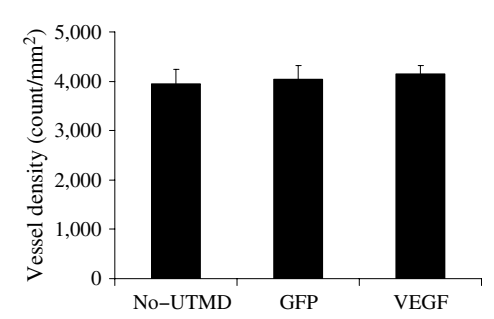

f Normal

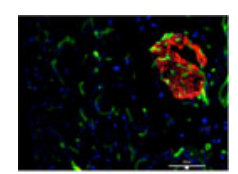

STZ

STZ

+ VEGF b
Normal liver

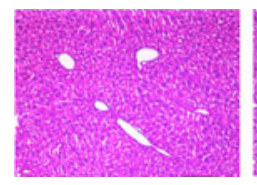

Day 2

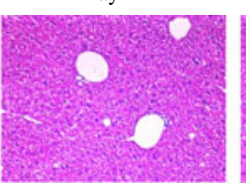

Day 7

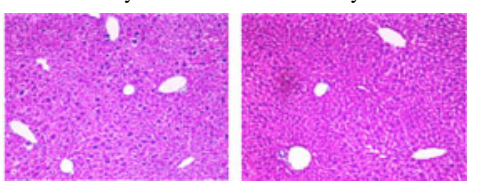

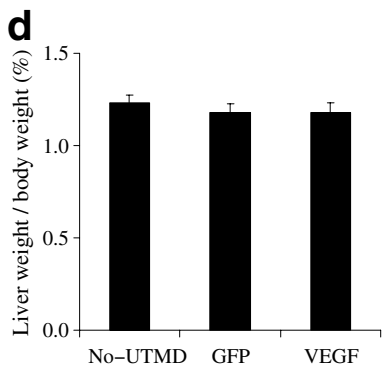

e

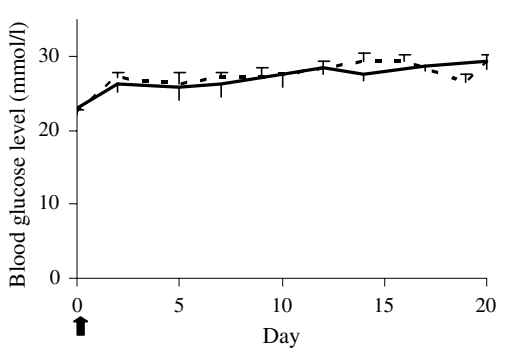

h

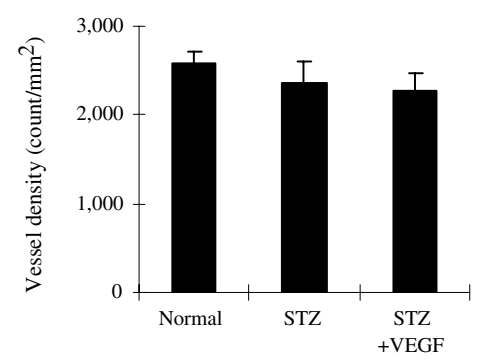

Fig. 3 a-d Effect of UTMD and hVEGF on liver. a Serum AST (solid line) and ALT (broken line) levels after UTMD. Arrow, time of UTMD treatment. b Histological analysis of liver after UTMD (haematoxylin and eosin staining). Original magnification $\times 100$. c Vessel density of liver at day 32 after treatment. There was no significant difference among the three groups. d The ratio of the weight of left lobe of liver to the body weight at day 32 after UTMD. There was no significant difference among the three groups. $\mathbf{e}-\mathbf{h}$ Effect of $V E G F$ on endogenous pancreas. $V E G F$ gene was delivered to liver of STZ-induced diabetic mice by UTMD (STZ+VEGF group). They were compared with non-treatment mice (normal group) and STZinduced diabetic mice (STZ group). e Non-fasting blood glucose levels after treatment in STZ (broken line) and STZ+VEGF (solid line) groups. Arrow, time of UTMD treatment. f Representative sections of pancreases stained for insulin, CD31 and DAPI at $\times 100$. Scale bar $100 \mu \mathrm{m}$. g Beta cell mass in pancreases at day 20 after treatment $(n=3)$. h Vessel density in islets at day 20 after treatment. $V E G F$ did not affect beta cell mass or vessel density. ${ }^{* *} p<0.01$ 
hVEGF production in and around graft islets In our procedure, islet transplantation and gene delivery by UTMD were performed consecutively through the same route (portal vein). Therefore, it was expected that the induced gene could be efficiently delivered to graft islets and the surrounded tissues. With immunohistochemistry, hVEGF was detected in the periphery and the surrounding tissues of the islets, confirming successful transfer and expression of the exogenous angiogenic gene, but not in the centre of islets (Fig. 4). Moreover, very few insulin-positive beta cells doubly produced hVEGF (Fig. 4a). Some of the glucagon-positive cells co-produced hVEGF (Fig. 4b), whereas there were many double-positive cells for hVEGF and vimentin (Fig. 4c). These data indicate that hVEGF was mostly produced by mesenchymal cells.

\section{Effect of induced hVEGF on islet function after transplantation}

The effect of hVEGF on the islet function after transplantation was determined in terms of daily blood glucose, human insulin and C-peptide levels at day32. At post-transplantation day7, nine out of $11(82 \%)$ mice in the hVEGF group became euglycaemic whereas three out of eight $(38 \%)$ in the noUTMD and four out of seven (57\%) in the GFP group became euglycaemic. However, most of the mice in the no-UTMD and GFP groups showed gradual increase in blood glucose level and recurrence of diabetes over 30 days (Fig. 5). Overall, eight out of $11(73 \%)$ mice in the VEGF group became persistently euglycaemic, whereas one out of eight $(13 \%)$ in the control and one out of seven (14\%) in the GFP group were euglycaemic at day30 (Fig. 5a-d). The euglycaemia rate of the VEGF group at day30 was significantly higher than the no-UTMD group (Table $1, p<0.05$ ). Kaplan-Meier estimates showed the graft survival rate of the VEGF group was significantly higher than the other two groups (Fig. 5e, $p<$ 0.05 in no-UTMD vs UTMD and GFP vs UTMD). The average serum human insulin and C-peptide levels in the VEGF group were significantly higher than in the no-UTMD group (Fig. 6: insulin, no-UTMD 16.6 $\pm 7.6 \mathrm{pmol} / \mathrm{l}$; GFP: $37.0 \pm 16.5 \mathrm{pmol} / \mathrm{l}$; VEGF: $108.9 \pm 26.4 \mathrm{pmol} / \mathrm{l} ; p=0.013$ in
no-UTMD vs VEGF. C-peptide, no-UTMD: $68.4 \pm$ $37.5 \mathrm{pmol} / \mathrm{l}$; GFP: $115.3 \pm 58.4 \mathrm{pmol} / \mathrm{l}$; VEGF: 791.5 \pm $230.5 \mathrm{pmol} / 1 ; p=0.022$ in no-UTMD vs VEGF).

Glucose tolerance test To further define the function of the transplanted islets, IPGTTs were performed at day 31 after transplantation. The $2 \mathrm{~h}$ blood glucose levels in both the noUTMD and GFP groups were $>11.1 \mathrm{mmol} / \mathrm{l}$, whereas the VEGF group showed a normal pattern (Fig. 5f).

Intra-islet vascularisation and beta cell mass At 32 days after treatment, capillary density in the transplanted islets was evaluated with immunohistochemical analysis (Fig. 7a-e). The islets were visualised with anti-insulin antibody, and the intra-islet vessels were stained using both anti-human CD31 and anti-mouse CD31 antibodies, since it has been reported that intra-islet endothelial cells contribute to revascularisation of transplanted islets as well as host endothelial cells $[15,16]$. In the VEGF mice, capillary density was significantly higher than in both of the control groups (Fig. 7e: no-UTMD, 169 \pm 36 ; GFP, 227 \pm 39 ; VEGF, $649 \pm 51$ count $/ \mathrm{mm}^{2}$, respectively; $p<0.0001$ in no-UTMD vs VEGF and GFP vs VEGF). The vessel density in the VEGF group clearly increased compared with the noUTMD group; however, it was still significantly lower than the natural human islets in pancreas (original islets; $1,215 \pm$ 86 count $/ \mathrm{mm}^{2}, p<0.00001$ in VEGF vs original islets). Beta cell mass in the left lobe of liver at day 32 after treatment was significantly greater in the VEGF group than other groups (Fig. 7f: no-UTMD, $0.11 \pm 0.01$; GFP, $0.13 \pm 0.02$; VEGF, $0.26 \pm 0.02$, respectively; $p<0.005$ in no-UTMD vs VEGF and GFP vs VEGF).

\section{Discussion}

Revascularisation to the transplanted islets is essential to improve their survival [17-21]. It was reported that pancreatic islet production of VEGF is critical for islet vascularisa-
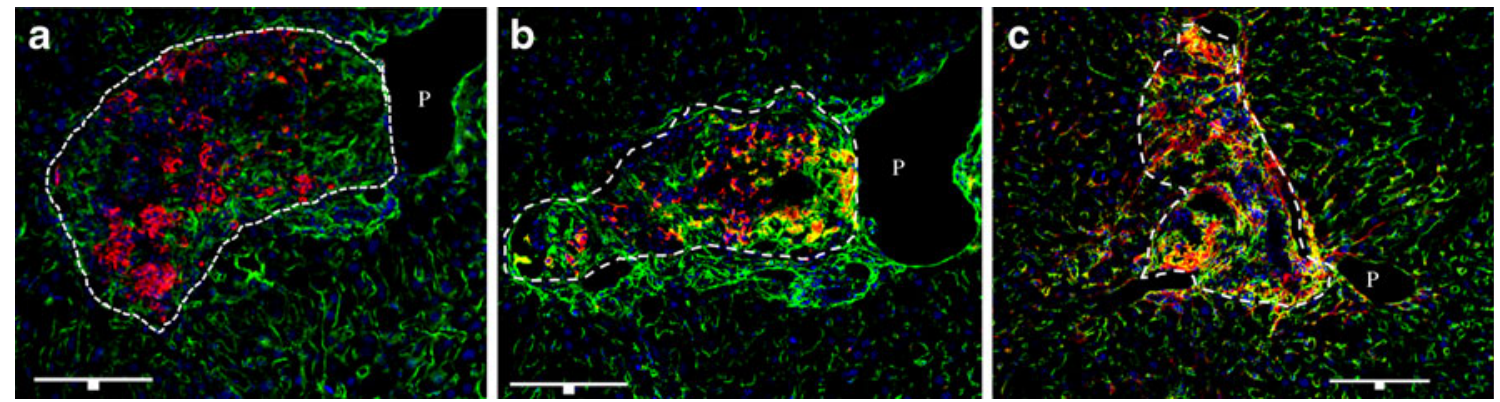

Fig. 4 hVEGF production in the graft islet and surrounding tissue after islet transplantation and UTMD. Immunohistochemistry of mouse liver 3 days after human islet transplantation followed by UTMD with VEGF. hVEGF was mostly detected in the surface and outer part of islets as well as the surrounding tissue. Green, hVEGF; red, human insulin (a), human glucagon (b), vimentin (c); blue, DAPI. Magnification $\times 200$, White line, border of graft islet; P, lumen of portal vein. Scale bar, $100 \mu \mathrm{m}$ 

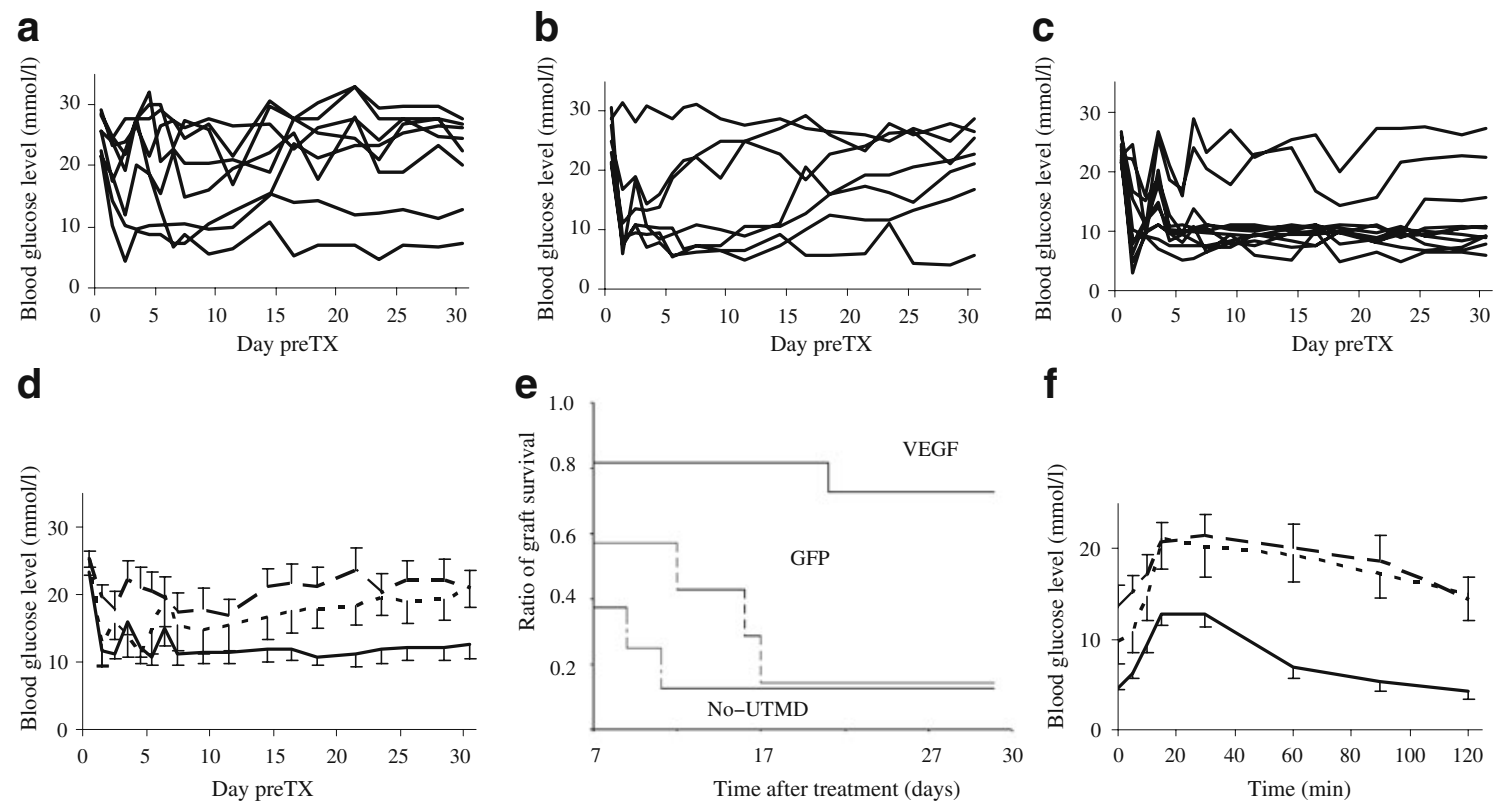

f

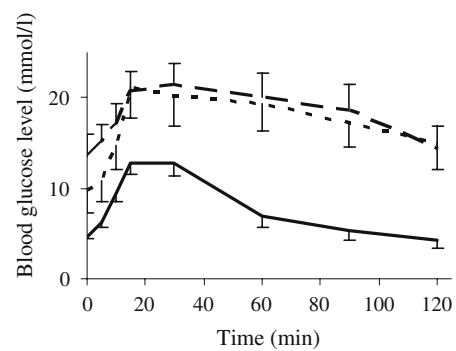

Fig. 5 a-d Blood glucose levels in the three groups. Five hundred human islets were transplanted into livers of STZ-induced diabetic nude mice. Then UTMD was performed with GFP (b: GFP group, $n=7$ ) or $V E G F$ (c: VEGF group, $n=11$ ) plasmid. UTMD was not conducted in the no-UTMD group (a: $n=8)$. d Average blood glucose levels in the three groups. Broken line, no-UTMD group; dotted line, GFP group; solid line, VEGF group. preTX, pretreatment. e Kaplan-Meier plot of

tion and function [22]. To promote the revascularisation of the transplanted islets, ex vivo transduction of islets with an adenoviral vector encoding $V E G F$ has been examined with evidence of revascularisation and improved islet survival [3, 23-25]. However, viral gene therapy is associated with severe adverse events [7, 8]. Hydrodynamics-based delivery of naked pDNA showed a therapeutic effect [24]; nevertheless its procedure is clinically unsuitable. It was shown that simple injection of pDNA alone without hydrodynamic pressure is ineffective [26]. At first, we tested the infusion of naked $V E G F$ plasmids via the portal vein without microbubbles and ultrasound because this is the simplest method. However, the gene expression was not detected in liver (data not shown). Then, we examined our developed UTMD

Table 1 The rate of euglycaemia 30 days after treatment in the three groups

\begin{tabular}{|c|c|c|c|}
\hline \multirow[t]{2}{*}{ Value } & \multicolumn{3}{|l|}{ Group } \\
\hline & no-UTMD & GFP & VEGF \\
\hline Transplant $(n)$ & 8 & 7 & 11 \\
\hline Euglycaemia $(n)$ & 1 & 1 & 8 \\
\hline Percentage & 12.5 & 14.3 & 72.7 \\
\hline
\end{tabular}

$p<0.05$ for no-UTMD vs VEGF by Tukey's honestly significant difference test graft survival of the three groups. Day 7 was set as a baseline and the $p$ value for the survival curve was determined by the logrank test. There were significant differences between the no-UTMD group (lower curve) and the VEGF group (top curve, $p<0.05$ ) and between the GFP group (middle curve) and the VEGF group $(p<0.05)$. $\mathbf{f}$ IPGTT at day 31 after treatment in the three groups. Broken line, no-UTMD group; dotted line, GFP group; solid line, VEGF group

method, which has been shown to be highly effective without significant adverse bioeffects [9-11, 27-30].

In this study, we employed a clinically relevant intraportal islet transplantation model. Our results provide evidence that the $V E G F$ gene can be targeted non-invasively to the liver and the transplanted islets, and can modify the intra-islet microvasculature. Notably, this is the first evidence that noninvasive delivery of a transgene to the host liver has therapeutic potential and it can produce biological changes in the vascularisation of graft islets. Intraportal injection of microbubbles has advantages. First, we could directly deliver a highly concentrated pDNA to liver compared with i.v. injection that was shown more effective than intrahepatic delivery [31]. Second, it could result in efficient gene
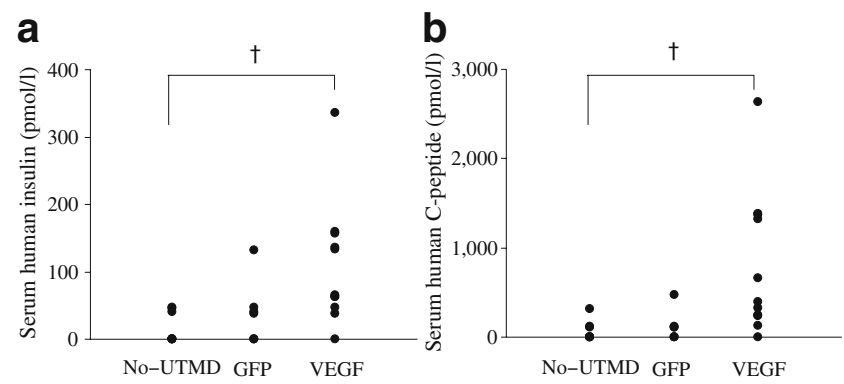

Fig. 6 Serum human insulin (a) and C-peptide levels (b) in the three groups. Blood was collected from each mouse at day 32 after treatment. ${ }^{\dagger} p<0.025$ 

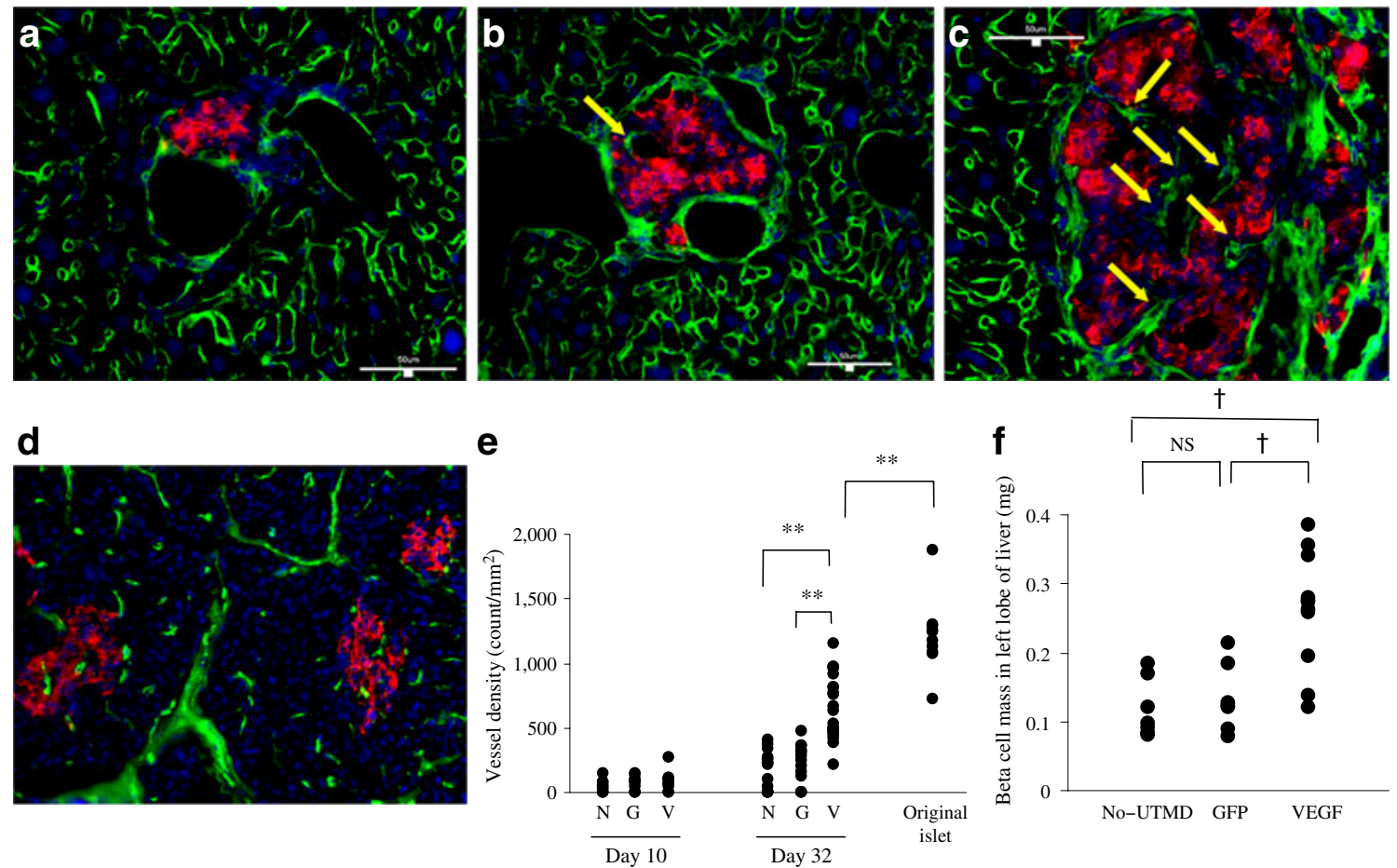

Fig. 7 Revascularisation of graft islets and beta cell mass at day 32 after treatment. a-d Representative sections stained for human insulin, CD31 and DAPI at $\times 200$ magnification. a no-UTMD group. b GFP group. c VEGF group. Several vessels were detected in the transplanted islet in the VEGF group. $\mathbf{d}$ Normal human islets in pancreas as a control. Red, human insulin; green, CD31; blue, DAPI; arrow, vessels in islets. e Vessel density in transplanted islets. Individual

values in the three groups for vessel density at day 10 and 32 after treatment are shown. Original human islets were used as a control. At day 32 , the vessel density of the VEGF group was significantly higher than both the no-UTMD and GFP group; however, it was significantly lower than the original islets. N, no-UTMD group; G, GFP group; V, VEGF group. $\mathbf{f}$ Beta cell mass in the left lobe of liver at day 32 after treatment. ${ }^{* *} p<0.01 ;{ }^{\dagger} p<0.005$

delivery to both the graft islet and the surrounding tissues. Obviously this could increase total gene expression compared with gene delivery only to islets, and it would be another advantage of UTMD over ex vivo gene delivery. For the purpose, we used CMV promoter instead of rat insulin promoter, which was shown to be effective in specifically directing genes to islets by UTMD. Although the results of this study indicated that the adverse effect of UTMD to liver was minimal, we should carefully assess hepatic toxicity in larger animals and ultimately in humans.

The increased microvasculature in graft islets probably contributed to the improvement of efficacy of islet transplantation. However, considering the time course of graft islet loss and $V E G F$ expression and the extent of revascularisation, there might be the possibility of another mechanism for the effect of $V E G F$ on the improved engraftment. Further studies would be required to reveal it.

In this study, the capillary density increased in the transplanted islets 32 days after $V E G F$ plasmid gene transfer. Nevertheless, induced hVEGF protein was detectable in the treated mice only for 14 days in blood and for 10 days in liver. We previously reported that VEGF delivery to heart in the rat by UTMD increased capillary density but a regression of capillary density to the baseline level was observed by day 30 , probably because of the transient nature of the plasmid expression [9]. We speculate that normal myocardium does not require an additional blood supply, so that the angiogenic vessels regress. In contrast, transplanted islets need a new blood supply, and 10-14 days of hVEGF stimulation might lead to further angiogenesis in transplanted islets. Since upregulated hVEGF production could lead to abnormal blood vessels and haemangiomas in islets [32], the transient $V E G F$ expression of our method can also be an advantage.

The effect of VEGF gene delivery by our method on the native pancreas was very minor and negligible as UTMD was targeted to the liver. Therefore, islet neogenesis in native pancreas does not explain the mechanisms of improved islet function.

Ultrasonic microbubble destruction causes cavitation, thermal effects, microstreaming, free radical production and microcapillary ruptures [33-36], which might activate endothelial cells or other cells. Indeed, slight inflammation and disruption of hepatocyte architecture were observed in both the GFP and VEGF groups (data not shown), although our data showed very little liver dysfunction after UTMD. Islet transplantation itself causes severe inflammation; therefore, the potential adverse bioeffects of microbubble 
destruction appear to be offset by the therapeutic effects of gene therapy in the present study. To prevent the inflammation associated with islet transplantation, a combined use of $V E G F$ and anti-inflammation genes or drugs might be useful [37].

In conclusion, VEGF gene delivery by UTMD to the transplanted islets and the host liver led to increasing vessel density in the graft islets and improving graft function. Gene delivery by UTMD is safe and effective in improving islet transplantation.

Acknowledgements This work was supported by NIH R01 HL072430-01 (P. A. Grayburn) and NIH 2P01 DK58398 (Newgard). This work was also partially supported by All Saints Health Foundation.

Duality of interest The authors declare that there is no duality of interest associated with this manuscript.

\section{References}

1. Ryan EA, Paty BW, Senior PA et al (2005) Five-year follow-up after clinical islet transplantation. Diabetes 54:2060-2069

2. Berney T, Ricordi C (2000) Islet cell transplantation: the future? Langenbecks Arch Surg 385:373-378

3. Narang AS, Sabek O, Gaber AO, Mahato RI (2006) Coexpression of VEGF and IL-1receptor antagonist improves human islet survival and function. Pharmaceutical Res 23:1970-1982

4. Fernandes JR, Duvivier-Kali VF, Keegan M et al (2004) Transplantation of islets transduced with CTLA4-Ig and TGF $\beta$ using adenovirus and lentivirus vectors. Transplant Immunology 13:191200

5. Rehman KK, Bertera S, Trucco M, Gambotto A, Robbins PD (2007) Immunomodulation by adenoviral-mediated SCD40-Ig gene therapy for mouse allogeneic islet transplantation. Transplantation 84:301-307

6. Panakanti R, Mahato RI (2009) Bipartite adenoviral vector encoding hHGF and hIL-1Ra for improved human islet transplantation. Pharm Res 26:587-596

7. Hacein-Bey-Abina S, von Kalle C, Schmidt M et al (2003) A serious adverse event after successful gene therapy for X-linked severe combined immunodeficiency. N Engl J Med 348:255-256

8. Manno CS, Pierce GF, Arruda VR et al (2006) Successful transduction of liver in hemophilia by AAV-Factor IX and limitations imposed by the host immune response. Nat Med 12:342-347

9. Korpanty G, Chen S, Shohet RV et al (2005) Targeting of VEGFmediated angiogenesis to rat myocardium using ultrasonic destruction of microbubbles. Gene Ther 12:1305-1312

10. Chen S, Ding JH, Bekeredjian R (2006) Efficient gene delivery to pancreatic islets with ultrasonic microbubble destruction technology. Proc Natl Acad Sci USA 103:8469-8474

11. Chen S, Ding J, Yu C (2007) Reversal of streptozotocin-induced diabetes in rats by gene therapy with betacellulin and pancreatic duodenal homeobox-1. Gene Ther 14:1102-1110

12. Matsumoto S, Noguchi H, Shimoda M et al (2010) Seven consecutive successful clinical islet isolations with pancreatic ductal injection. Cell Transplant. doi:10.3727/096368909X481773

13. Matsumoto S, Okitsu T, Iwanaga Y et al (2006) Successful islet transplantation from non-heart-beating donor pancreata using modified Ricordi islet isolation method. Transplantation 82:460465

14. Montaña E, Bonner-Weir S, Weir GC (1993) Beta cell mass and growth after syngeneic islet cell transplantation in normal and streptozocin diabetic C57BL/6 mice. J Clin Invest 91:780787

15. Linn T, Schneider K, Hammes HP et al (2003) Angiogenic capacity of endothelial cells in islets of Langerhans. FASEB J 17:881883

16. Brissova M, Fowler M, Wiebe P (2004) Intraislet endothelial cells contribute to revascularization of transplanted pancreatic islets. Diabetes 53:1318-1325

17. Narang AS, Mahato RI (2006) Biological and biomaterial approaches for improved islet transplantation. Pharmacol Rev 58:194-243

18. Lau J, Carlsson PO (2009) Low revascularization of human islets when experimentally transplanted into the liver. Transplantation $87: 322-325$

19. Jones GL, Juszczak MT, Hughes SJ, Kooner P, Powis SH, Press M (2007) Time course and quantification of pancreatic islet revascularization following intraportal transplantation. Cell Transplant 16:505-516

20. Menger MD, Vajkoczy P, Beger C, Messmer K (1994) Orientation of microvascular blood flow in pancreatic islet isografts. J Clin Invest 93:2280-2285

21. Vajkoczy P, Olofsson AM, Lehr HA (1995) Histogenesis and ultrastructure of pancreatic islet graft microvasculature. Evidence for graft revascularization by endothelial cells of host origin. Am J Pathol 146:1397-1405

22. Brissova M, Shostak A, Shiota M (2006) Pancreatic islet production of vascular endothelial growth factor-A is essential for islet vascularization, revascularization, and function. Diabetes 55:2974-2985

23. Narang AS, Cheng K, Henry J (2004) Vascular endothelial growth factor gene delivery for revascularization in transplanted human islets. Pharm Res 21:15-25

24. Cheng K, Fraga D, Zhang C (2004) Adenovirus-based vascular endothelial growth factor gene delivery to human pancreatic islets. Gene Ther 11:1105-1116

25. Zhang N, Richter A, Suriawinata J et al (2004) Elevated vascular endothelial growth factor production in islets improves islet graft vascularization. Diabetes 53:963-970

26. Miao CH, Thompson AR, Loeb K, Ye X (2001) Long-term and therapeutic-level hepatic gene expression of human factor IX after naked plasmid transfer in vivo. Mol Ther 3:947-957

27. Bekeredjian R, Chen S, Pan W, Grayburn PA, Shohet RV (2004) Effects of ultrasound-targeted microbubble destruction on cardiac gene expression. Ultrasound Med Biol 30:539-543

28. Bekeredjian R, Chen S, Frenkel PA, Grayburn PA, Shohet RV (2003) Ultrasound-targeted microbubble destruction can repeatedly direct highly specific plasmid expression to the heart. Circulation 108:10221026

29. Chen S, Shohet RV, Bekeredjian R, Frenkel P, Grayburn PA (2003) Optimization of ultrasound parameters for cardiac gene delivery of adenoviral or plasmid deoxyribonucleic acid by ultrasound-targeted microbubble destruction. J Am Coll Cardiol 42:301-308

30. Chen S, Kroll MH, Shohet RV, Frenkel P, Mayer SA, Grayburn PA (2002) Bioeffects of myocardial contrast microbubble destruction by echocardiography. Echocardiography 19:495500

31. Shen ZP, Brayman AA, Chen L, Miao CH (2008) Ultrasound with microbubbles enhances gene expression of plasmid DNA in the liver via intraportal delivery. Gene Ther 15:1147-1155 
32. Ozawa CR, Banfi A, Glazer NL (2004) Microenvironmental VEGF concentration, not total dose, determines a threshold between normal and aberrant angiogenesis. J Clin Invest 113:516527

33. Miller DL, Gies RA (1998) The interaction of ultrasonic heating and cavitation in vascular bioeffects on mouse intestine. Ultrasound Med Biol 24:123-128

34. Wu J, Ross JP, Chiu JF (2002) Reparable sonoporation generated by microstreaming. J Acoust Soc Am 111:1460-1464
35. Kondo T, Misík V, Riesz P (1998) Effect of gas-containing microspheres and echo contrast agents on free radical formation by ultrasound. Free Radic Biol Med 25:605-612

36. Skyba DM, Price RJ, Linka AZ, Skalak TC, Kaul S (1998) Direct in vivo visualization of intravascular destruction of microbubbles by ultrasound and its local effects on tissue. Circulation 98:290-293

37. Panakanti R, Mahato RI (2009) Bipartite vector encoding hVEGF and hIL-1Ra for ex vivo transduction into human islets. Mol Pharm 6:274-284 